\title{
Player's Model: Criteria for a Gameplay Profile Measure
}

\author{
Emmanuel Guardiola and Stephane Natkin \\ CEDRIC /CNAM, 292 Rue Saint-Martin \\ 75003 Paris, France \\ emmanuel.guardiola@seasideagency.com, stephane.natkin@cnam.fr
}

\begin{abstract}
Game designers empirically use psychological and sociological player's model to create the gameplay of their video games. These models are generally implicit and always informal. A formal analysis of the player's model leads to define efficient player behavior profile. It can have numerous applications, for instance adaptation of the content to the player's ability and interest. Our work tries to find a rational way to assess Players Styles, concept suggested by Bartle [1] in 1996. The first step, state of the art of the player model, shows already some interesting criteria that can be used to classify player's styles.
\end{abstract}

Keywords: Video games, player's model, player's profile, gameplay, game design.

\section{Introduction: The Playing Player}

Bartle was the first designer trying to analyze the player's behavior from a gameplay point of view. His classification of players in MUD (Multi User Dungeon) is still a reference discussed both by designers and sociologist. Bartle's model is based on interviews done after game sessions. A methodological potential flaw is related to the fact that data were collected out of the magic circle [4]. As a consequence any measurement should investigate the specific playing time, when the player feels engaged [2] and perceives his posture as an active player [3]. So the measure has to be performed during active gameplay.

The main criticism regarding Bartle's model is related to contextual evaluation of the player's behavior. It can only reveal MUD players' profiles for a given game and at a given period. So the player model may be built on general criteria already used to create all sort of game and more generally based on general behavioral theories.

Our goal is to analyze criteria needed to assess the player while he's playing and reacting to gameplay mechanisms. Therefore we should rely on a definition of Gameplay.

Gameplay definition: Gameplay is often defined as the relation between player and game world as Salen and Zimmerman evoke it [5]. On one hand we have a designed game system that simulates something. On the other hand, we have a player, creating personal mental imagery of the situation and deciding mentally how to act within. Between the game system and the player, interfaces and captors create the link. In the 
gameplay situations, sometimes called patterns [6], the player has a goal, a kind of challenge to reach that goal while facing uncertainty about how the game will end [7].

Therefore, the definition of gameplay used in this research should be: All actions (cognitive or physical, performances and strategies) performed by the player, influencing negatively or positively the outcome of the uncertain game situation in which he is immersed in.

\section{Player Model in Gameplay State of Art}

Model of the playing player can be explored from several empirical and academic points of view. This paper focuses on the constructive aspects of the harvesting.

Empirical difficulty and Flow. Game developers spend important resources in design, test and tuning to insure that the player is always confronted to an interesting challenge, not boring and not too frustrating. All these expenses seem to be legitimized by Milahy Csikszentmihalyi theory of flow [8]. Difficulty test sessions include a model of the player built upon pragmatic aspects of his gameplay: how much time he takes to end a level, number of "game over" in a level... From data collected, developers design the path through their games for a generic player or several classes of players if the game offers several levels of difficulty. Some games, as Oblivion [9], implement a dynamic difficulty system linked to the player's performance in the game.

Two criteria help measuring the relationship between goal and effective result: Efficiency and Effectiveness. Efficiency is: how much resources are spent to reach the player's goal. For example, many "Survival horror" are fully based on the quality of resources management (rare ammunitions, inventory). Effectiveness is: does the player reach the goal (or how long did it take to reach the goal)? Another possible criterion to be measured in test lab is perseverance: How many times a player retries a given challenge? Does the player focus on one quest or micro challenge until he succeeds or does he try to find alternatives?

Empirical critical path and bonus. In almost all game levels or even challenges there are several ways to win. Some are based on the "longest path": In most solo action adventure games, first person shooter, role playing games, designers create a critical path: A walkthrough across all the levels that allows the player to meet with most of the assets produced. But, if the player rushes he cans win using a "shortest path". As a consequence he missed potential bonus, hidden rewards and secret levels. Some players want to end the game, others want to see, know or get everything. An evaluation of the ratio of the number of events, situations, challenges encountered by the player using his strategy and the "longest past" ones can be considered as characteristics of player's behavior. This is, in Bartle's classification difference between Killers and Explorers, for example.

Performance modeling. All games, according to Jesper Juul's definition, include a scoring system. A scoring system is generally a poor indicator of the player's behavior but it can be enhanced in several ways. In the game Forza Motorsport [10] 
the Drivatar feature is an efficient way to model the player's performance [11]. As he drives the player trains an AI. The system records a few key characteristics linked to gameplay as how consistent you are in, your speed and line or at what point you brake before entering a turn. Then you can let the AI drives a car with some of your gameplay attributes. This research shows spectacularly that cloning gameplay performance is possible with a very few variables to track.

Artificial intelligence. Artificial intelligence research gives several points of view on the player's model. The goal can be, for instance, to adapt challenge to the player or create more human behavior for non-player's character.

Historically in Chess and Go AI, the modeling process target is to create a virtual player who can find the optimal solution in a challenge. But real players are not always optimal in their choices.

Another approach is based on tracing the strategies used in specific contexts. It allows for creation of an Action Model with identification of strategy through gameplay patterns. All gameplay's variables must be taken in account. This is easy to do with games like Pacman [12] or Space invader [13] which rely only on a few features. It may be much more complex to realize on elaborates gameplay and in particular on sandbox games. The pattern recognition principle is mainly applicable to a priori defined strategies. How could a computer classify strategies that were not forecasted by the designer?

If strategies preferences are good criteria to model the player, we need a complementary way to capture player's style.

Decision theory. Decision Theory provides an interesting behavioral model of decision under risk, [14]. This scientific model matches with a classification of poker players used by players themselves to guess competitors' strategies: Risk-seeking for gains of low probability, Risk-aversion for losses of low probability, Risk-aversion for gains of high probability, Risk-seeking for losses of high probability. Customizing that approach for video game, we may classify this behavior in a two dimension scheme by measuring the percentage of success of challenges together with player involvement in it.

Two other notions linked to Decision Theory can be taken into account and added to our Gameplay criteria. The Utility principle: $5 \$$ do not have the same value for everybody. Players evaluate subjectively the value of the resources available and potential rewards. The ambiguity principle: we take decision with a certain amount of information on the situation, a number of known variables. What amount is acceptable for us?

Motivation Theory. Guillaume Denis [16] uses the motivation continuum of Deci and Ryan [17] in video game context. We can identify player progression from amotivation to intrinsic motivation. Role playing games as World of Warcraft (WOW) [18] provide several layer of motivational content to help the player progression through different levels of external regulations (See table 1). At the integrated regulation level, we can identify Bartle's player classification. 
Game Theory. In a previous work, we used game theory to analyze gameplay decision [15]. A game situation can be sorted out in a finite number of matrices. We can track player choices and try to define the pay off. The conclusion of this experiment reveals a possible way to differentiate short, medium and long term behaviors.

Table 1. Deci and Ryan motivation continuum table with WOW regulation progression

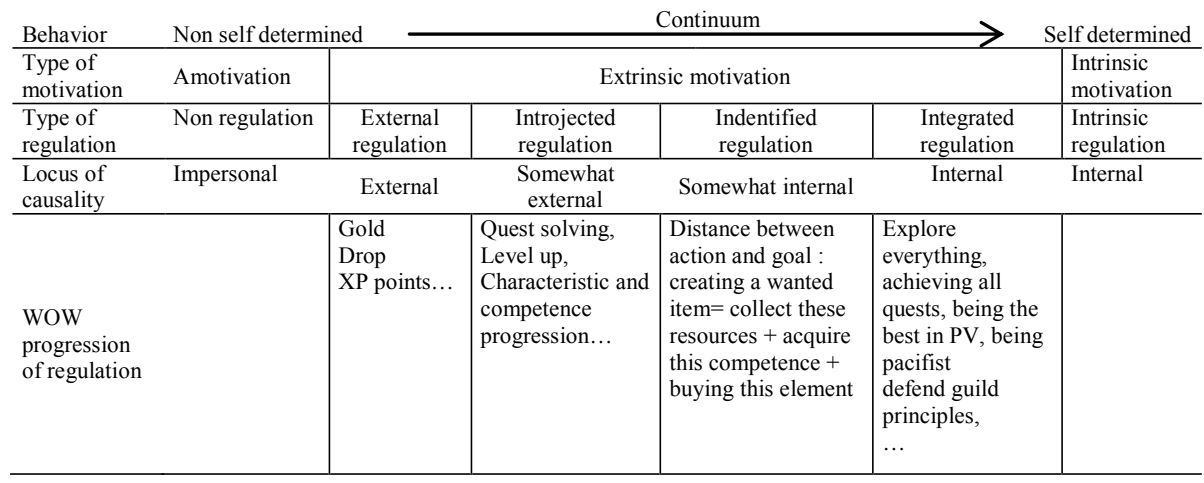

\section{Criteria of the Player Gameplay Profile}

From the previous section, we can identify several interesting criteria to establish a player's gameplay profile.

Table 2. Criteria for a gameplay profile

\begin{tabular}{|l|l|}
\hline Performance & In this particular gameplay, how the player performs? \\
\hline Efficiency & How much resources I spend to reach the goal. \\
\hline Effectiveness & Do I reach the goal? \\
\hline Strategies preferences & $\begin{array}{l}\text { What type of strategies the player knows, uses, masters. What } \\
\text { context/strategies pattern he reproduces }\end{array}$ \\
\hline Completion & What \% of the reachable game he finished \\
\hline Perseverance & What is his degree of retrying facing failure? \\
\hline Risk aversion profile & What is the position of the player in the risk aversion area? \\
\hline Utility & What is his level of utility, what are the valuables elements for him? \\
\hline Ambiguity & Does he take risk in uncertainty or knowing all the variables? \\
\hline Motivation type & Where is the player on the motivation continuum? \\
\hline $\begin{array}{l}\text { Pay off term } \\
\text { preference }\end{array}$ & Is he short-term, medium-term or long term in his choices? \\
\hline
\end{tabular}

The most pragmatic trace of player's decision is the input. This information must be interpreted through the filter of the game system to understand its gameplay meaning. We work with the company Mimesis Republic on their virtual playground Mamba Nation [19] to confront this list to real data records. To be efficient, criteria were transformed in dimensions linked to clear player input. This work brings out several remarks. 
Performance measure must be elaborated for each game, and in the game, for each gameplay. Creating an average performance for the all game can reduce the precision of the result. Strategies preference and risk aversion profile are also to be assessed for each gameplay. These three criteria need to analyze with attention the game system to define the gameplay pattern.

Efficiency and effectiveness assessment with precision requires clarifying what is the player's objective. In sand box games we need a layer of interpretation to define the objective.

Completion is the percentage of game completed within reachable content. The interactive objects and interaction potentials must be clearly defined for each game. It can be mapped to discover goals, weapon to use, a collection to complete, a quest to activate...The player must know it all.

Ambiguity needs to be assessed to measure what the player knows of a situation. The difficulty here is to understand how he perceives information. We can eventually trace all the critical elements displayed to him by sound, rumble and images. Even by doing so, we cannot be sure of his perception and understanding.

Transforming Motivation localization in measurable token in the game needs layers of interpretation and can create bias in the result. The simple fact you open a shop interface can mean the player perceives an expensive object that can drive his gameplay choices for a long period of time. Pay-off term preference is in the same case. For these two criteria the way we build the interpretation had to be exhaustively documented and communicated to the final user of the player profiling tools.

Some criteria can be measured with temporality influences as an historic or by mixing other parameters: performance progression through time, strategic preference of people at noon and during the evening.

\section{Conclusion}

The criteria table is the draft of an individual gameplay player profile grid. It can be adapted to several types of games. This plasticity is a progression from previous behavioral approach. We do not impose types; records of a population may reveal specific behaviors for a specific game.

Our next step will be to embody the grid in a game and perform real time recordings.

\section{References}

1. Bartle, R.A.: Hearts, Clubs, Diamonds, Spades: Players who suit MUDs. Journal of MUD research 1 (1996); reprint in Salen, K., Zimmerman, E. (ed.) The Game Design Reader. MIT Press, Cambridge (2006)

2. Juul, J.: Half-real. MIT Press, Cambridge (2005)

3. Henriot, J.: Sous couleur de jouer. Jose Corti Publisher, Paris (1989)

4. Huizinga, J.: Homo ludens, Essai sur la fonction sociale du jeu. Gallimard, Paris (1951)

5. Salen, K., Zimmerman, E.: Rule of Play: Game Design Fundamentals. MIT Press, London (2004)

6. Koster, R.: Theory of fun for game design. Paraglyph Press, Scottsdale (2005) 
7. Guardiola, E.: Ecrire pour le jeu. Dixit, Paris (2000)

8. Csikszentmihalyi, M.: Flow: The Psychology of Optimal Experience. Harper Perennial, New York (1991)

9. Bethesda: The Elder Scrolls IV Oblivion. 2K Games Publisher (2006)

10. Microsoft game studios: Forza Motorsport. Microsoft Game Studios Publisher (2005)

11. Herbrich, R., Graepel, T.: Playing machines: machine learning application in video games, http://research.microsoft.com/mlp/Forza/FMD_manual.htm?0sr=a, Applied game group, Microsoft Research Cambridge,

http://research.microsoft.com/

en-us/projects/drivatar/forza.aspx

12. Namco: Pacman (1979)

13. Taito: Space Invader (1978)

14. Tversky, A., Kahneman, D.: Advances in Prospect Theory: Cumulative Representation of Uncertainty. Journal of Risk and Uncertainty 5, 297-323 (1992)

15. Guardiola, E., Natkin, S.: Game Theory and video game, a new approach of game theory to analyze and conceive game systems. In: CGAMES 2005, Angouleme (2005)

16. Denis, G.: Jeux vidéo éducatifs et motivation: application à l'enseignement du jazz, Thèse à l'Ecole des mines de Paris (2006)

17. Deci, E.L., Ryan, R.M.: The "What" and "Why" of Goal Pursuits: Human Needs and the Self-Determination of Behavior. Psychological Inquiry 11, 227-268 (2000)

18. Blizzard: World of Warcraft. Vivendi Universal Publisher (2004)

19. Mimesis Republic: Mamba Nation, in development. Mimesis Republic Publisher (2010) 